\title{
An integrated approach to active model adaptation and on-line dynamic optimisation of batch processes
}

\author{
Tao Chen ${ }^{\mathrm{a}}$, Yi Liu ${ }^{\mathrm{b}}$, Junghui Chen ${ }^{\mathrm{c}, *}$ \\ ${ }^{a}$ Department of Chemical and Process Engineering, University of Surrey, Guildford, GU2 7XH, UK \\ ${ }^{b}$ Institute of Process Equipment and Control Engineering, Zhejiang University of Technology, \\ Hangzhou, 310032, China \\ ${ }^{c}$ Department of Chemical Engineering, Chung-Yuan Christian University, Chung-Li, Taiwan 320, \\ Republic of China
}

\begin{abstract}
In the application of on-line, dynamic process optimisation, adaptive estimation of the system states and parameters is usually needed to minimise the unavoidable model-process mismatch. This work presents an integrated approach to optimal model adaptation and dynamic optimisation, with specific focus on batch processes. An active approach is proposed whereby the input variables are designed so as to maximise the information content of the data for optimal model adaptation. Then, this active adaptation method is combined with the objective of process performance to form a multi-objective optimisation problem. This integrative approach is in contrast to the traditional adaptation method, where only the process performance is considered and adaptation is passively carried out by using the data as is. Two strategies for solving the multi-objective problem are investigated: weighted average and constrained optimisation, and the latter is recommended for the ease in determining the balance between these two objectives. The proposed methodology is demonstrated on a simulated semi-batch fermentation process.
\end{abstract}

Keywords: Adaptive filtering, Closed-loop identification, Design of experiments, Moving horizon estimation, Multi-objective optimisation, Parameter estimation

\footnotetext{
*Corresponding author, Tel.: +886 3-265-4107.

Email addresses: t.chen@surrey.ac.uk (Tao Chen), jason@wavenet.cycu.edu.tw (Junghui Chen)
} 


\section{Introduction}

Model-based dynamic optimisation is an established technology to facilitate safe, economic and environmentally benign operation of manufacturing processes, particularly for those operating in batch or semi-batch modes. The basic principle of dynamic process optimisation is to formulate a model-predicted performance measure, which is then optimised to give the desired time trajectory of the input variables (for example, the trajectory of temperature or flow rate until the end of batch). In theory, dynamic optimisation can be carried out off-line, and the pre-calculated input trajectory can be implemented on-line by using a conventional regulatory controller. However, this feed-forward approach relies too much on the availability of high-quality model, and is too sensitive to model-process mismatch. This mismatch is not completely avoidable because of, for instance, lack of understanding of process mechanisms, use of simplifying assumptions, measurement errors, and continuous change of process behaviour due to, for example, catalyst deactivation and heat exchanger fouling. Therefore, a more desired approach is on-line optimisation: the optimisation problem is repeatedly solved during the process operation, when new measurements are collected and used to update the model and its prediction $[1,2,3]$. This feed-back feature of on-line dynamic optimisation is similar to that of model predictive control, though the latter normally focuses on optimally tracking a certain pre-computed trajectory. Because of this similarity, the following analysis of existing studies in the literature does not stress the difference between on-line optimisation and control unless necessary.

A key task of dynamic optimisation is the on-line adaptation of the model by using new measurements so as to minimise the model-process mismatch. This adaptation stage can be active and "open-loop": by suspending the process optimiser, the input variables can be purposely designed to collect the maximal information for model update. For batch processes, the method of optimal design of experiments (DoE) $[4,5]$ has ample algorithms to serve this purpose, such as the $A$-optimal and $D$-optimal designs. Alternatively, adaptation can be passive and "closed-loop": the model is updated whilst 
the inputs are still determined by the process optimiser. Typical methods for updating mechanistic models include extended Kalman filters, particle filters, moving horizon estimation (MHE), among others $[6,7,8,9,10]$. However, both approaches have flaws. In the absence of process optimiser, open-loop adaptation interrupts the normal process operation and is costly due to additional experimental resources, off-spec products, and sometimes even safety violations. Conversely, closed-loop adaptation is convenient and maintains the usual operation; yet it lacks the capability of adjusting inputs so as to attain the data that are optimal for model adaptation.

As a consequence, integration of active and closed-loop model adaptation has become a desired choice, giving rise to the emergence of closed-loop identification. Nevertheless, previous work was primarily focused on adapting linear models for continuous processes around steady-states $[11,12,13]$. In contrast, batch processes usually require highly non-linear models and do not have steady-states. In addition, very limited effort was devoted to the optimal design of inputs; notable methods include adding random "dithering" signal to inputs [14], spectral analysis [13], and persistent excitation [11]. These approaches to the design of inputs are also limited to linear continuous processes around steady-states. Similar idea to combine process optimisation/control with optimal model adaptation was reflected in dual control methods, which have mainly been applied to, again, linear continuous processes $[15,16,17]$. It was recognised that dual control is potentially useful for non-linear batch processes [18]; however, to the best knowledge of the authors, this has not been explored.

The primary aim of this paper is to develop an integrative approach to simultaneous active model adaptation and dynamic process optimisation. This approach may be viewed as a hybrid route whereby the inputs are designed through a trade-off between optimal model adaptation and optimal process operation. We will extend the concept of such integration from within linear continuous systems to complex batch processes. From another perspective, the present study may also be viewed as extending the offline integration of process optimisation and parameter estimation $[19,20,21]$ to on-line applications. Such integration may also be regarded as a trade-off between exploration 
(of the decision space) and exploitation (of the model): exploration aims to improve the model by collecting the most informative data, whilst exploitation is to rely the current model for decision making.

The rest of this paper is organised as follows. Section 2 gives the detailed formulation of the integrated dynamic optimisation and active model adaptation problem. The solution strategy will also be discussed, including three components: (i) the design of objective function for optimal process operation, (ii) the design of objective function for optimal model parameter adaptation, and (iii) MHE for the update of system states and parameters. The proposed methodology will be demonstrated in Section 3 using a simulated fed-batch bioreactor that is well known in the literature for studying control and optimisation algorithms [20]. Finally, concluding remarks are given in Section 4.

\section{Problem formulation and solution strategy}

Consider the following generic state-space model for a batch process:

$$
\begin{aligned}
\mathbf{x}_{t+1} & =\mathbf{f}\left(\mathbf{x}_{t}, \mathbf{p}, \mathbf{u}_{t}\right)+\mathbf{w}_{t} \\
\mathbf{y}_{t} & =\mathbf{h}\left(\mathbf{x}_{t}, \mathbf{p}\right)+\mathbf{v}_{t}
\end{aligned}
$$

where $\mathbf{x}$ is the state vector, $\mathbf{y}$ is the measurement vector, and $t$ denotes the time index. The state function $\mathbf{f}(\cdot)$ describes the system dynamics that depends on the state at time $t$, the model parameters $\mathbf{p}$, and the input variables $\mathbf{u}_{t}$ (also termed manipulated variables). The process noise $\left(\mathbf{w}_{t}\right)$ is utilised to describe the random mismatch between the model and the actual process. The measurement is a function $(\mathbf{h}(\cdot))$ of states and parameters subject to noise $\mathbf{v}_{t}$. Note that the noise terms $\left(\mathbf{w}_{t}\right.$ and $\mathbf{v}_{t}$ ) are assumed additive, following the convention in process control. In addition, if no specific information is available, both noise terms are assumed to be independent and identically distributed (i.i.d.) according to a multivariate normal distribution: $p_{w}\left(\mathbf{w}_{t}\right)=N(\mathbf{0}, \mathbf{Q}), p_{v}\left(\mathbf{v}_{t}\right)=N(\mathbf{0}, \mathbf{R})$, where $p$ denotes the probability distribution function $(p d f)$. The common practice is to restrict $\mathbf{Q}$ and $\mathbf{R}$ to diagonal matrices. The 
measurement noise covariance $\mathbf{R}$ is usually derived from the precision properties of measurement devices. The covariance matrix for the process noise needs to be either tuned or estimated in order to infer the system states. A variety of methods have been reported to address this issue [22, 23, 24].

Given the process model, the proposed approach iterates between on-line dynamic optimisation, and model adaptation through state and parameter estimation. The flow chart is illustrated in Figure 1. The detailed solution methods are presented in the rest of this section.

\subsection{On-line dynamic optimisation}

The basic concept of on-line dynamic optimisation, similar to that of model predictive control, is to repeatedly (at each time step) solve an open-loop optimal control problem subject to the system dynamics and the constraints for states and inputs. For batch processes, the usual performance measure is a function of the end-batch (time $F)$ states and parameters $\psi\left[\mathbf{x}_{F}, \mathbf{p}\right]$ :

$$
\min _{\mathbf{u}_{t}, \ldots, \mathbf{u}_{F}} J=\min _{\mathbf{u}_{t}, \ldots, \mathbf{u}_{F}}\left\{\psi\left[\mathbf{x}_{F}, \mathbf{p}\right]+\lambda \phi\left[\mathbf{u}_{t}, \ldots, \mathbf{u}_{F}\right]\right\}
$$

subject to $\quad \mathbf{g}\left(\{\mathbf{x}, \mathbf{u}\}_{t, \ldots, F}, \mathbf{p}\right) \leq 0$

and the process model in eqs. (1)(2)

where $t$ is the current time and $\lambda$ is a weighting factor to penalise a certain function of the inputs. This function, $\phi$, can be used to discourage the abrupt change of the inputs or the deviation from a certain nominal input profile. Without loss of generality, we set $\lambda=0$ in this study and focus on the end-batch process performance, which needs to be predicted by solving the system model from the current estimate of states $\mathbf{x}_{t}$ and parameters $\mathbf{p}$. The state and parameter estimation problem will be discussed in detail in Section 2.3. Other objective functions can also be designed in order to, for example, track a certain reference state trajectory. The constraints include those imposed on functions of the states, inputs and model parameters $(\mathbf{g}(\cdot))$, as well as the state space 
model (eqs. (1)(2)). The inputs are usually parameterised to be piecewise constant or linear with respect to time.

\subsection{On-line optimisation with active model adaptation}

It is well understood from off-line process optimisation studies [19, 20, 21] that the optimal inputs in terms of process performance is often not optimal in the sense of adapting model parameters to changing process characteristics. As a result, simply accepting the measurements that are produced by the process optimisation-oriented inputs is undesired for model adaptation. Instead, an active approach should be considered to generate data that contain more information for model update. The measure of data information can be borrowed from the literature of DoE, such as the Fisher information matrix $\mathbf{F}$ given below:

$$
\mathbf{F}=\int_{t}^{F}\left(\frac{\partial \mathbf{y}_{\tau}}{\partial \mathbf{p}}\right)^{T} \mathbf{R}\left(\frac{\partial \mathbf{y}_{\tau}}{\partial \mathbf{p}}\right) d \tau
$$

where the derivative of the measurement $\left(\mathbf{y}_{\tau}\right)$ with respect to the model parameter $\mathbf{p}$ at time $\tau$ is still a function of $\mathbf{p}$, and it should be evaluated at the current estimate $\mathbf{p}^{*}$. The derivative can be obtained using the following relation:

$$
\frac{\partial \mathbf{y}}{\partial \mathbf{p}}=\frac{\partial \mathbf{h}}{\partial \mathbf{p}}+\frac{\partial \mathbf{h}}{\partial \mathbf{x}} \frac{\partial \mathbf{x}}{\partial \mathbf{p}}
$$

where $\partial \mathbf{x} / \partial \mathbf{p}$ also varies with time:

$$
\frac{d}{d t}\left(\frac{\partial \mathbf{x}}{\partial \mathbf{p}}\right)=\frac{\partial \mathbf{f}}{\partial \mathbf{x}} \frac{\partial \mathbf{x}}{\partial \mathbf{p}}+\frac{\partial \mathbf{f}}{\partial \mathbf{p}}
$$

which can be solved along with the ordinary differential equations representing the process (eq. (1) is the corresponding discrete form).

Therefore, in order to produce data that are more informative for parameter estimation, a certain scalar function of $\mathbf{F}$ should be optimised. The usual choices include (but are not limited to) [25]: 
- A-criterion (average criterion): $\min \left[\operatorname{Tr}\left(\mathbf{F}^{-1}\right)\right]$. This is to minimise the average parameter estimation error, or equivalently to maximise the trace of $\mathbf{F}$.

- E-criterion (eigenvalue criterion): $\max \left[\lambda_{\min }(\mathbf{F})\right]$. This is to maximise the smallest eigenvalue of $\mathbf{F}$, or equivalently to minimise the largest parameter error whilst ignoring other parameters.

- Modified E-criterion: $\min \left[\lambda_{\max }(\mathbf{F}) / \lambda_{\min }(\mathbf{F})\right]=\min [\sigma(\mathbf{F})]$. This ME-criterion is to minimise the condition number of the Fisher information matrix, $\sigma(\mathbf{F})$, with known optimum of unity.

Notably, different criteria have different aims and there are no consensus as to which is the most appropriate in practice. For example, the A-criterion considers the aggregate effect of all parameters but may be insensitive to the most uncertain parameter, which, in contrast, is of exclusive interest to the E-criterion. In this study, we adopt the ME-criterion that may provide a good balance between the most and least uncertain parameters.

Following the formulation of optimal process operation and optimal model adaptation, the overall problem becomes multi-objective optimisation that seeks to minimise $J$ in eq. (3) and $\sigma(\mathbf{F})$ simultaneously. Given the possible conflict between objectives, the aim of multi-objective optimisation is to find the set of Pareto (non-dominant) solutions. Standard methods are: weighted aveage, converting all but one criteria into constraints, and evolutionary algorithms [26]. Nevertheless, in the context of automatic process operation, presenting the full set of Pareto points to a decision maker is not a viable approach. Instead, a simple weighted average is typically utilised [20]:

$$
\min _{\mathbf{u}_{t}, \ldots, \mathbf{u}_{F}}[(1-\alpha) J+\alpha \sigma(\mathbf{F})]
$$

A major challenge of this weighted objective function is the selection of $\alpha$. Suppose that the process performance measure, $J$, is the end-batch production in the unit of grams, whilst the condition number is usually in the order of $10^{6}$ or even larger. It is 
extremely difficult to determine an appropriate $\alpha$ prior to the operation of an actual batch. If the multi-objective problem is converted into constrained optimisation, it is also difficult to determine the bound for either $J$ or $\sigma(\mathbf{F})$.

In the present work, we propose a two-step solution. First, the process performance $J$ is optimised alone, without considering the objective for optimal parameter estimation. Given this optimum $\hat{J}$, the second step is to solve the following constrained optimisation problem:

$$
\min _{\mathbf{u}_{t}, \ldots, \mathbf{u}_{F}}[\sigma(\mathbf{F})], \quad \text { subject to } \quad J<\hat{J}+(1-\beta)\left(J_{\text {worst }}-\hat{J}\right)
$$

where $J_{\text {worst }}$ is the worst (i.e. maximal) possible value for $J$, and thus $\left(J_{\max }-\hat{J}\right)$ defines the range of performance degradation. Therefore, $(1-\beta)$ may be intepreted as performance sacrifice that we are willing to tolerate so that to obtain better parameter estimate. Although $\beta$ is still a user-determined term, its selection is much more intuitive. For example, $\beta=0.99$ might be a reasonable choice; it represents a possible $1 \%$ performance degradation, leaving certain freedom to explore the process inputs that are more informative for parameter estimation. In some practical situations where the performance objective is to maximise the end-batch production, $J_{\text {worst }}$ could be set to 0 (no product produced), as in the case study presented in Section 3.

An alternative to the proposed approach in eq. (8), still in two steps, is to optimise the information content of the data first (i.e. $\hat{\sigma}(\mathbf{F})=\min \sigma(\mathbf{F})$ ), and then optimise the process performance (i.e. $\min J$ ) subject to an allowable maximal sacrifice on information content. However, the best information content could mean very poor process performance (c.f. the case study in Figure 3 when $\alpha$ is close to 1). It is not clear how much sacrifice on information content should be allowed to obtain good process performance. In addition, it is practically easier to determine the allowable sacrifice on process performance in exchange for better parameter estimation, since optimal process performance is the ultimate goal. Therefore, this alternative method is not explored further.

The use of both the weighted average and the two-step approach will be further 
discussed in the case study. Next, we outline the MHE algorithm that is used for state and parameter estimation.

\subsection{State and parameter estimation using $M H E$}

The estimation of states and (or) parameters in a dynamic system is usually cast into a filtering problem, whereby the well-known Kalman filter is an optimal solution if the state function is linear and the states are normally distributed [7]. When these assumptions do not hold, approximate methods need to be utilised, such as the extended Kalman filters, unscented Kalman filters [27, 28] and more recently particle filters $[6,29,10]$. These filters are sequential in the sense that they infer the system states at the current time step by utilising the current measurement only. The past information is implicitly included via the use of prior distribution for the states. As such, these filters are efficient in terms of computation, which may be a necessity for industrial process optimisation and control. However, these afore-mentioned filters are not directly applicable to constrained state estimation problems (e.g. states must be non-negative).

In contrast, MHE $[9,10]$ formulates the state and (or) parameter estimation problem over a finite time horizon, making use of the information from multiple time steps in the past. The state constraints can be explicitly incorporated in MHE by devising a constrained optimisation problem. Specifically, at time step $t$, MHE attempts to solve the following problem over a time horizon of $h$ steps (from $t-h+1$ to $t$ ):

$$
\min _{\mathbf{x}_{t-h+1}, \ldots, \mathbf{x}_{t}, \mathbf{p}}\left\{V\left(\mathbf{x}_{t-h+1}\right)+\sum_{j=t-h+1}^{t-1} L_{w}\left(\mathbf{w}_{j}\right)+\sum_{j=t-h+1}^{t} L_{v}\left(\mathbf{y}_{j}-\mathbf{h}\left(\mathbf{x}_{j}, \mathbf{p}\right)\right)\right\}
$$

subject to $\mathbf{x}_{j+1}=\mathbf{f}\left(\mathbf{x}_{j}, \mathbf{p}, \mathbf{u}_{j}\right)+\mathbf{w}_{j}$ in addition to other constraints (if any) on the states and parameters. In the above formula, the first term summarises the information about $\mathbf{x}_{t-h+1}$ obtained from the past up to time $t-h$. Rigorously, $V\left(\mathbf{x}_{t-h+1}\right)$ is the negative probability of $\mathbf{x}_{t-h+1}$ conditional on the measurement sequence $\left\{\mathbf{y}_{1}, \ldots, \mathbf{y}_{t-h}\right\}$. In the terminology of MHE, $V(\cdot)$ is called "arrival cost" and may be approximated by using 
an extended Kalman filter $[9,10] . L_{w}$ and $L_{v}$ are the negative log-likelihood functions, respectively: $L_{w}(\mathbf{w})=-\log p_{w}(\mathbf{w}), L_{v}(\mathbf{v})=-\log p_{v}(\mathbf{v})$, where $p_{w}$ and $p_{v}$ are the $p d f$ for process and measurement noises discussed previously. In particular, a normal distributed process noise, $p_{w}(\mathbf{w})=N(\mathbf{0}, \mathbf{Q})$, means

$$
L_{w}(\mathbf{w})=\frac{d_{w}}{2}(2 \pi)+\frac{1}{2} \operatorname{det}(\mathbf{Q})+\frac{1}{2} \mathbf{w}^{T} \mathbf{Q}^{-1} \mathbf{w}
$$

where $d_{w}$ is the dimension of $\mathbf{w}$; similar expression can be obtained for the process noise $p d f p_{v}(\mathbf{v})=N(\mathbf{0}, \mathbf{R})$. Note that apart from the current states and parameters, MHE also obtains a sequence of "smoothed" past states from time $t-h+1$ to $t-1$. These smoothed past states, although not used for the purpose of predictive optimisation and control, may be important information for post-analysis of the dynamic systems.

The solution to the optimisation problem in eq. (9) provides the estimate of current system states, which are required to optimise the predicted process objective function. The performance of MHE depends on the choice of the time horizon, $h$. In theory, longer time horizon carries more measurements and thus should give more accurate estimation. However, the dimension of the optimisation problem is $\left(h \times d_{x}+d_{p}\right)$ where $d_{x}$ and $d_{p}$ are the dimensions of the states and parameters, respectively. High dimensional optimisation tends to suffer from the issue of local optima, and thus in practice it may not always give better results than using a small $h$.

\section{Case study}

\subsection{The fed-batch fermentation process}

The simulated well-mixed fed-batch bioreactor [20] used in this study has been well studied in various applications, in particular in experimental design [30, 31]. The process dynamic behaviour is described by the following set of differential equations: 


$$
\begin{aligned}
\frac{d S}{d t} & =-\sigma X+\frac{u}{V}\left(S_{i n}-S\right) \\
\frac{d X}{d t} & =\mu X-\frac{u}{V} X \\
\frac{d V}{d t} & =u
\end{aligned}
$$

where $u(\mathrm{~L} / \mathrm{h})$ is the feed flow rate of substrate; the state variables and their initial values at the beginning of a batch are given in Table 1 . The specific growth rate is of the Haldane type:

$$
\mu=\frac{\mu_{m} S}{K_{p}+S+S^{2} / K_{i}}
$$

with three parameters, $\mu_{m}, K_{p}$ and $K_{i}$. The substrate consumption rate is given by

$$
\sigma=\frac{\mu}{Y}+m
$$

where the yield $Y$ and maintenance factor $m$ are assumed to be fixed. The objective of the process is to maximise the biomass production, $V_{F} X_{F}$, at the end of the batch.

All parameter values are given in Table 2. For the purpose of illustrating the proposed methodology, two parameters, $K_{p}$ and $K_{i}$, are assumed to be unknown and will be adapted during dynamic optimisation of the batch process. Some initial guess of both parameters is used at the beginning of the process. To simulate parameter discrepancy, the true parameter values are multiplied by a factor $r$ to give the initial guess, where for simplicity the same $r$ value is applied to both $K_{p}$ and $K_{i}$. A range of $r$ will be tested to explore the impact of the severity of such parameter discrepancy on the performance of process optimisation and model adaptation: $r \in\{0.5,1.0,1.5,2.0,2.5,3.0\}$, $r=1.0$ meaning no initial mismatch in parameters. Estimation of $K_{p}$ and $K_{i}$ is a typical scenario to investigate the trade-off between optimal process operation and model adaptation. Eq. (14) suggests the importance of these two parameters in optimal process performance, because the maximal specific growth rate is achieved when $S=\sqrt{K_{p} K_{i}}$. Usually, an intermediate value of $S$ is desired to encourage biomass 
production. However, as far as parameter estimation is concerned, a small value of $S$ is preferred for the estimation of $K_{p}$ (because $\mu$ is sensitive to the change in $K_{p}$ ) but not for that of $K_{i}$, whilst a large $S$ is helpful for the estimation of $K_{i}$ but not $K_{p}$. Such conflict of goals requires multi-objective optimisation as described in the pervious methodology section.

(Tables 1 and 2 about here)

The batch duration, $F$, is fixed to $40 \mathrm{~h}$, and the sampling (measurement) interval is $4 \mathrm{~h}$. This long sampling time was chosen because in many industrial fermentation processes, the substrate concentration is only measured a few hours apart, through physical sampling and off-line analysis. The feed rate $u$ is constrained to be no more than $1 \mathrm{~L} / \mathrm{h}$. We assume that only two out of three states, i.e. the substrate concentration $S$ and reactor volume $V$, are directly measurable at each sampling interval. In simulation, the state and measurement noises are assumed to be independent and identically distributed, following zero mean Gaussian with diagonal covariance matrix: $\operatorname{diag}[\mathbf{Q}]=[0.01,0.01,0.01]^{2}$ and $\operatorname{diag}[\mathbf{R}]=[0.1,0.05]^{2}$; the former is meant to introduce a certain level of random model-process mismatch whilst the latter reflects typical noise in the measurements. The time horizon for MHE is fixed to four sampling steps (i.e. $16 \mathrm{~h}$ ), which was found in prelimary runs to be adequate whilst keeping computation reasonable. More details about the process can be found elsewhere [20]. The integrated model adaptation and optimisation were carried out at every sampling time.

Direct dynamic optimisation with respect to $\left\{u_{t}, \ldots, u_{F}\right\}$ is computationally expensive due to the high dimension of the input sequence. Unlike predictive control for tracking reference trajectory, the challenge originates from the need to predict endbatch performance, and thus optimisation of input from current time $t$ to the end of batch. In addition, high dimentional optimisation often suffers from the difficulty in finding (near) global optimum, even if advanced algorithms like multiple shooting are used. Instead, we adopt an indirect approach to constrain the form of the feed rate profile, following the strategy used by Versyck and van Impe [20]. In particular, a 
fixed optimal "set-point" for the substrate concentration, $\bar{S}$, is sought such that the objective function is optimised. Note that although $\bar{S}$ is fixed at each time step, it may have different values when moving from one time step to the next during which the optimisation problem is re-solved. This on-line optimisation approach accounts for the feed-back from the actual process behaviour, and it is in contrast to the off-line optimisation reported in [20]. Given $\bar{S}$, the feed rate at time $t\left(\right.$ i.e. $\left.S_{t}\right)$ is determined by a simple "on-off" strategy according to the following three scenarios:

1. $S_{t}>\bar{S}+\epsilon$ : no substrate is supplied, so that existing substrate can be consumed until the set-point is reached.

2. $S_{t}<\bar{S}-\epsilon$ : substrate is supplied at the maximum feed rate $(1 \mathrm{~L} / \mathrm{h})$, in order to bring $S$ to the set-point as soon as possible.

3. $S_{t} \in[\bar{S}-\epsilon, \bar{S}+\epsilon]$ : the feed rate is given by the following control law to keep the substrate concentration constant: $u=\sigma X V /\left(S_{i n}-\hat{S}\right)$.

Here a tolerance factor, $\epsilon$, is chosen to be 0.05 so as to avoid drastic change of feed rate whilst fairly tracking the set-point. The feed rate is parameterised as piece-wise constant within each "optimisation horizon", which is chosen to be $0.05 \mathrm{~h}$, giving rise to negligible discretisation error in terms of realising the optimal set-point $\bar{S}$. Direct parameterisation of $u$ to such small time interval would result in a very high dimensional optimisation problem. This "on-off" strategy, though being simple, is still widely used in fermentation and other industries for batch manufacturing $[32,20]$.

The overall substrate feed is also constrained by availability, which is set to $3 \mathrm{~L}$. Ideally, the entire substrate will be fed before the end of batch and fully utilised. However, we have adopted a fixed batch duration of $40 \mathrm{~h}$, which may be justified by ease of scheduling when compared with variable batch duration.

\subsection{Results and discussions}

A typical process trajectory is illustrated in Figure 2 without any initial parameter discrepancy $(r=1.0)$. The substrate feed rate was determined by dynamic optimisation, only maximising the end-batch biomass production. Thus the figure shows 
a typical batch with high performance: the production was $327.7 \mathrm{~g}$. Although the biomass concentration $(X)$ was not directly measured, MHE was quite effective in estimating it from the substrate concentration $(S)$ and liquid volume $(V)$. The two parameters were also fairly followed, except for small mismatch towards the end of the batch (Figure 2(b)). The feed rate profile is also typical for a well performing bioprocess [20]: all substrate was fed into the reactor and consumed by the end of the batch. Nevertheless, the results in this figure were obtained without any parametric model-process mismatch; only a small amount of random distrubance was introduced as state noise $\mathbf{w}_{t}$. Next, we explore the effectiveness of the proposed active adaptation approach when subjecting to various degrees of parameter discrepancy.

(Figure 2 about here)

Figure 3 summarises the end-batch biomass production $V_{F} X_{F}$ when using the weighted objective function in eq. (7) for dynamic optimisation, as a function of the weighting factor $\alpha$. Note that $\alpha=0$ means no active adaptation, and the feed rate was solely designed to maximise $V_{F} X_{F}$. On the other side, $\alpha=1$ corresponds to no consideration of production and the only objective was to minimise the ME-criterion for optimal parameter estimation. Although the logrithm $\alpha$ axis does not allow the illustration of $\alpha=0$, the result from $\alpha=10^{-20}$ was verified to be numerically equivalent. The results confirm that the combination of process performance and data information is potentially useful: when $\alpha$ moves from $10^{-20}$ to until $10^{-3}$, the production is improved in some cases. To be specific, Table 3 lists the baseline $\left(\alpha=10^{-20}\right)$ and the best production. This improvement can be attributed to better and more rapid update of the model parameters so as to rectify the misleading model predictions as early as possible. To verify this hypothesis, Figures 4 and 5 compare the process profile and state/parameter estimation between the baseline $\left(\alpha=10^{-20}\right)$ and best $\left(\alpha=10^{-5}\right)$ cases when $r=0.5$. The noticeable spikes in the feed rate are due to the use of the on-off operation strategy, and they would be avoided should a filtering method be used (e.g. the simple moving average filter). Clearly, the parameter estimates adapted to 
the actual values more quickly when active adaptation was included in the objective function. As a result, the prediction of the end-batch production was more accurate by using active adaptation, as illustrated in panel (d) of both figures. Note that panel (d) compares the predicted and the "actual" final production, should the entire input sequence be applied until the end of the batch. In on-line dynamic optimisation, the input sequence is applied only until the next sampling time, and the optimal control problem is re-solved; the re-designed input sequence thus results in a different "actual" production at each time step.

(Figures 4 and 5 about here)

(Table 3 about here)

Another observatin from Figure 3 and Table 3 is that the method of active adaptation and optimisation is more needed when model-process mismatch becomes larger. As expected, when no initial parameter mismatch is present, the proposed active adaptation does not give significant improvement, nor does it deteriorate the process significantly until $\alpha>10^{-8}$. However, when the initial parameter guess is three times of the true values $(r=3.0)$, the production can be increased from $179.5 \mathrm{~g}$ to $282.7 \mathrm{~g}$, i.e. an improvement of $57.4 \%$. Nevertheless, these results also highlight the major challenge when applying the weighting strategy in practice: how to determine the optimal weight $\alpha$ ? It may be natural to avoid the values close to unity, since this would place too little emphasis on maximising production, as manifested in Figure 3. However, even some small values, e.g. $\alpha=10^{-12}$ in Figure 3(c) and $\alpha=10^{-10}$ in Figure 3(d), resulted in dramatically reduced production. It seems that the objective function does not vary smoothly with $\alpha$, especially in the presence of large parameter discrepancy. In addition, the optimal $\alpha$ varies significantly from case to case. Such difficulty mainly lies in the lack of physical meaning of the Fisher information matrix's condition number, which was used to measure the information content of the data for model update. The values of production and condition number are different in several orders of magnitude, making the determination of an appropriate weight extremely problematic. 
To address the above issue regarding the choice of the weight, we further explore the two-step constrained optimisation strategy, formulated in eq. (8). Figure 6 summarises the production as a function of $\beta, 1-\beta$ being the fraction of performance sacrifice that can be tolerated in the hope to obtain better parameter estimate. Intuitively, $\beta$ should be chosen to be close to unity, and this is consistent with the results in Figure 6. Focusing on the region where $\beta \geq 0.9$, the detailed results are given in Table 4 . In four out of the six cases (except $r=0.5$ and $r=1.0$ ) with varying parametric discrepancy, the maximal improvement is better than that obtained from weighted approach. More importantly, the choice of $\beta$, though still remaining a subjective matter, is much easier than that of $\alpha$. Although it is still not possible to identify the best $\beta$ a priori, it appears that a value greater than 0.95 would give practically useful improvement over the baseline case. Nevertheless, the optimal value of $\beta$ clearly depends on the magnitude of model-process mismatch. When such mismatch is very significant like in the case of $r=3.0$, a smaller value of $\beta(0.95)$ appears to be helpful to allow more emphasis on the information content of the data, giving rise to more rapid adaptation of the model to the process.

(Figure 6 about here)

(Table 4 about here)

\section{Concluding remarks}

This paper presents an active approach to model adaptation in conjunction with on-line dynamic optimisation, with particular interest in batch processes. The input variables are designed to optimise two often conflicting objectives: to maximise the information content of the data for optimal model adaptation, and to optimise the defined process performance. This integrative approach is in contrast to the traditional adaptation method, where only the process performance is considered and adaptation is passively carried out by using the data as is. Two strategies for solving the multi-objective problem are explored: weighted average and constrained optimisation. 
Through demonstration on a simulated fed-batch fermentation process, we recommend the two-step constrained optimisation approach, because it is easier to determine an appropriate balance between these two objectives. The presented work was focused on parametric mismatch between the model and the process. If the model significantly deviates from the process because of structural mismatch (e.g. important process phenomena not modelled), then more advanced adaptation methods are needed [33], and accordingly the proposed active approach will need to be re-designed. This aspect requires more follow-on studies.

The proposed methodology can be further refined. For example, it may be desired to monitor the model-process mismatch by using the estimated parameter uncertainty, based on which the tuning factor $\beta$ in the constrained optimisation can be adjusted on-line. If the uncertainty is high, then $\beta$ should be set to slightly away from unity, reflecting the emphasis on updating the model. Conversely, $\beta$ can be set to very close to unity, when the model-process mismatch becomes negligible, to allow more focus on the process performance. Such an approach is currently under investigation.

\section{Acknowledgement}

The authors acknowledge financial support for this work from R\&D Center for Membrane Technology, Chung-Yuan Christian University, Taiwan, Republic of China.

[1] J. S. Alford, Bioprocess control: Advances and challenges, Computers and Chemical Engineering 30 (2006) 1464-1475.

[2] D. Bonvin, Optimal operation of batch reactors: a personal view, Journal of Process Control 8 (1998) 355-368.

[3] C. Kiparissides, P. Seferlis, G. Mourikas, A. J. Morris, Online optimizing control of molecular weight properties in batch free-radical polymerization reactors, Industrial and Engineering Chemistry Research 41 (2002) 6120-6131. 
[4] P. Englezos, N. Kalogerakis, Applied Parameter Estimation for Chemical Engineers, Marcel Dekker, 2001.

[5] G. Franceschini, S. Macchietto, Model-based design of experiments for parameter precision: State of the art, Chemical Engineering Science 63 (19) (2008) 48464872.

[6] T. Chen, J. Morris, E. Martin, Particle filters for state and parameter estimation in batch processes, Journal of Process Control 15 (2005) 665-673.

[7] A. H. Jazwinski, Stochastic Processes and Filtering Theory, Academic, New York, 1970.

[8] Y. Liu, W. Chen, H. Wang, Z. Gao, P. Li, Adaptive control of nonlinear timevarying processes using selective recursive kernel learning method, Industrial and Engineering Chemistry Research 50 (5) (2011) 2773-2780.

[9] C. Rao, J. Rawlings, Constrained process monitoring: Moving-horizon approach, AIChE Journal 48 (2002) 97-109.

[10] J. Rawlings, B. Bakshi, Particle filtering and moving horizon estimation, Computers and chemical engineering 30 (10-12) (2006) 1529-1541.

[11] H. Genceli, M. Nikolaou, New approach to constrained predictive control with simultaneous model identification, AIChE Journal 42 (10) (1996) 2857-2868.

[12] N. D. Pour, B. Huang, S. L. Shah, Consistency of noise covariance estimation in joint input-output closed-loop subspace identification with application in LQG benchmarking, Journal of Process Control 19 (10) (2009) 1649-1657.

[13] Y. Zhu, P. van den Bosch, Optimal closed-loop identification test design for internal model control, Automatica 36 (8) (2000) 1237-1241.

[14] C. Koung, J. MacGregor, Identification for robust multivariable control - the design of experiments, Automatica 30 (10) (1994) 1541-1554. 
[15] N. Filatov, H. Unbehauen, Survey of adaptive dual control methods, IEE Proceedings - Control Theory and Applications 147 (1) (2000) 118-128.

[16] D. Li, F. Qian, P. Fu, Variance minimization approach for a class of dual control problems, IEEE Transactions on Automatic Control 47 (12) (2002) 2010-2020.

[17] D. Li, F. Qian, P. Fu, Optimal nominal dual control for discrete-time linearquadratic Gaussian problems with unknown parameters, Automatica 44 (1) (2008) $119-127$.

[18] J. Zhang, A reliable neural network model based optimal control strategy for a batch polymerization reactor, Industrial and Engineering Chemistry Research 43 (2004) 1030-1038.

[19] J. Chen, D. Wong, S. Jang, S. Yang, Product and process development using artificial neural-network model and information analysis, AIChE Journal 44 (1998) $876-887$.

[20] K. Versyck, J. van Impe, Feed rate optimization for fed-batch bioreactors: from optimal process performance to optimal parameter estimation, Chemical Engineering Communications 172 (1999) 107-124.

[21] W. Yan, S. Hu, Y. Yang, F. Gao, T. Chen, Bayesian migration of Gaussian process regression for rapid process modeling and optimization, Chemical Engineering Journal 166 (2011) 1095-1103.

[22] G. Leu, R. Baratti, An extended Kalman filtering approach with a criterion to set its tuning parameters; application to a catalytic reactor, Computers and Chemical Engineering 23 (2000) 1839-1849.

[23] D. Loebis, R. Sutton, J. Chudley, W. Naeem, Adaptive tuning of a Kalman filter via fuzzy logic for an intelligent AUV navigation system, Control Engineering Practice 12 (2004) 1531-1539. 
[24] J. Valappil, C. Georgakis, Systematic estimation of state noise statistics for extended Kalman filters, AIChE Journal 46 (2000) 292-308.

[25] E. Walter, L. Pronzato, Identification of Parametric Models from Experimental Data, Springer, Paris, 1997.

[26] K. Deb, Multi-objective optimization using evolutionary algorithms, John Wiley and Sons, 2001.

[27] S. J. Julier, J. K. Uhlmann, Unscented filtering and nonlinear estimation, Proceedings of the IEEE 92 (2004) 401-422.

[28] A. Romanenko, J. Castro, The unscented filter as an alternative to the EKF for nonlinear state estimation: a simulation case study, Computers and Chemical Engineering 28 (2004) 347-355.

[29] T. Chen, J. Morris, E. Martin, Dynamic data rectification using particle filters, Computers and Chemical Engineering 32 (2008) 451-462.

[30] A. M. Cappuyns, K. Bernaerts, I. Y. Smets, O. Ona, E. Prinsen, J. Vanderleyden, J. F. Van Impe, Optimal fed batch experiment design for estimation of monod kinetics of azospirillumbrasilense: From theory to practice, Biotechnology Progress 23 (5) (2007) 1074-1081.

[31] R. Schenkendorf, A. Kremling, M. Mangold, Optimal experimental design with the sigma point method, IET Systems Biology 3 (2009) 10-23.

[32] C. Birol, C. Ündey, A. Cinar, A modular simulation package for fed-batch fermentation: penicillin production, Computers and Chemical Engineering 26 (2002) 1553-1565.

[33] B. Chachuat, B. Srinivasan, D. Bonvin, Adaptation strategies for real-time optimization, Computers and Chemical Engineering 33 (10) (2009) 1557 - 1567. 


\section{List of Tables}

$1 \quad$ System states. . . . . . . . . . . . . . . . . . . . 23

2 Model parameters. . . . . . . . . . . . . . . . . . 24

3 End-batch production (g) by optimising the weighted objective function. The value of $\alpha$ for the baseline case is $10^{-20} \ldots \ldots$. . . . . . . . 25

4 End-batch production (g) by the two-step approach to optimising the constrained objective function. The baseline case corresponds to $\beta=1$, and it is the basis for calculating the relative improvement. The maximal production under each case is in boldface. The production worse than the baseline is illustrated in brackets. . . . . . . . . . . . . . . . . .

\section{List of Figures}

1 The flow chart of the overall approach. . . . . . . . . . . . . . 27

2 The process trajectory and state/parameter estimation results for the case $r=1.0$, i.e. no initial parameter discrepancy. The weighted objective function with $\alpha=10^{-20}$ was used for optimisation, numerically equivalent to no active adaptation. The end-batch production was 327.7 g. (a) Substrate $(S)$ and biomass $(X)$ concentration; (b) Parameter estimation; (c) Reactor volume and feed rate. . . . . . . . . . . . .

3 The end-batch biomass production $V_{F} X_{F}$ as a function of the weighting factor $\alpha$, subjecting to initial parameter discrepancy in terms of the multiplier $r$ : (a) $r=0.5$, (b) $r=1.0$, (c) $r=1.5$, (d) $r=2.0$, (e) $r=2.5$, (f) $r=3.0 \ldots \ldots \ldots \ldots \ldots \ldots$ 
4 The process trajectory and state/parameter estimation results for the case $r=0.5$. The weighted objective function with $\alpha=10^{-20}$ was used for optimisation, numerically equivalent to no active adaptation. The end-batch production was 294.0 g. (a) Substrate $(S)$ and biomass $(X)$ concentration; (b) Parameter estimation; (c) Reactor volume and feed rate; d) Predicted end-batch production at each sampling interval. . . .

5 The process trajectory and state/parameter estimation results for the case $r=0.5$. The weighted objective function with $\alpha=10^{-5}$ was used for optimisation, and the end-batch production was 334.9 g. (a) Substrate $(S)$ and biomass $(X)$ concentration; (b) Parameter estimation; (c) Reactor volume and feed rate; (d) Predicted end-batch production at each sampling interval. . . . . . . . . . . . . . . 31

6 The end-batch biomass production $V_{F} X_{F}$ as a function of the constraint fraction $\beta$, subjecting to initial parameter discrepancy in terms of the multiplier $r$ : (a) $r=0.5$, (b) $r=1.0$, (c) $r=1.5$, (d) $r=2.0$, (e) $r=2.5$, (f) $r=3.0 \ldots \ldots \ldots \ldots \ldots \ldots$ 
Table 1: System states.

\begin{tabular}{lll}
\hline State & Description & Initial value \\
\hline$S(\mathrm{~g} / \mathrm{L})$ & Substrate concentration & 0 \\
$X(\mathrm{~g} / \mathrm{L})$ & Biomass concentration & 1.5 \\
$V(\mathrm{~L})$ & Bioreactor volume & 7 \\
\hline
\end{tabular}


Table 2: Model parameters.

\begin{tabular}{lll}
\hline Parameter & Description & Nominal value \\
\hline$\mu_{m}\left(\mathrm{~h}^{-1}\right)$ & Maximal specific growth rate & 2.1 \\
$K_{p}(\mathrm{~g} / \mathrm{L})$ & Half-saturation constant & 50 \\
$K_{i}(\mathrm{~g} / \mathrm{L})$ & Substrate inhibition constant & 0.5 \\
$Y(\mathrm{~g} / \mathrm{g})$ & Yield & 0.47 \\
$m(\mathrm{~g} / \mathrm{g})$ & Maintenance factor & 0.29 \\
$S_{i n}(\mathrm{~g} / \mathrm{L})$ & Feed substrate concentration & 500 \\
$F(\mathrm{~h})$ & Batch duration & 40 \\
\hline
\end{tabular}


Table 3: End-batch production (g) by optimising the weighted objective function. The value of $\alpha$ for the baseline case is $10^{-20}$.

\begin{tabular}{lllll}
\hline$r$ & Baseline & Best & Best $\alpha$ & Improvement \\
\hline 0.5 & 294.0 & 334.9 & $10^{-5}$ & $13.9 \%$ \\
1.0 & 327.7 & 331.3 & $10^{-15}$ & $1.1 \%$ \\
1.5 & 311.3 & 322.5 & $10^{-8}$ & $3.6 \%$ \\
2.0 & 306.7 & 306.7 & $10^{-20}$ & $0.0 \%$ \\
2.5 & 231.7 & 289.6 & $10^{-5}$ & $25.0 \%$ \\
3.0 & 179.5 & 282.7 & $10^{-3}$ & $57.4 \%$ \\
\hline
\end{tabular}


Table 4: End-batch production (g) by the two-step approach to optimising the constrained objective function. The baseline case corresponds to $\beta=1$, and it is the basis for calculating the relative improvement. The maximal production under each case is in boldface. The production worse than the baseline is illustrated in brackets.

\begin{tabular}{lllllll}
\hline$r$ & $\beta$ & & & & & \\
& 1 & 0.995 & 0.99 & 0.95 & 0.9 & Max improvement \\
\hline 0.5 & 294.0 & 315.8 & $\mathbf{3 2 5 . 1}$ & 305.1 & $(253.6)$ & $10.6 \%$ \\
1.0 & 327.7 & $\mathbf{3 2 8 . 7}$ & $(326.6)$ & $(313.3)$ & $(294.1)$ & $0.3 \%$ \\
1.5 & 311.3 & $\mathbf{3 2 7 . 7}$ & 320.4 & 313.1 & $(278.4)$ & $5.2 \%$ \\
2.0 & 306.7 & 314.1 & $\mathbf{3 3 4 . 0}$ & 311.0 & $(280.2)$ & $8.9 \%$ \\
2.5 & 231.7 & $\mathbf{3 2 0 . 2}$ & 289.5 & 285.8 & 252.3 & $38.2 \%$ \\
3.0 & 179.5 & 248.4 & 282.0 & $\mathbf{3 0 0 . 9}$ & 288.1 & $67.6 \%$ \\
\hline
\end{tabular}




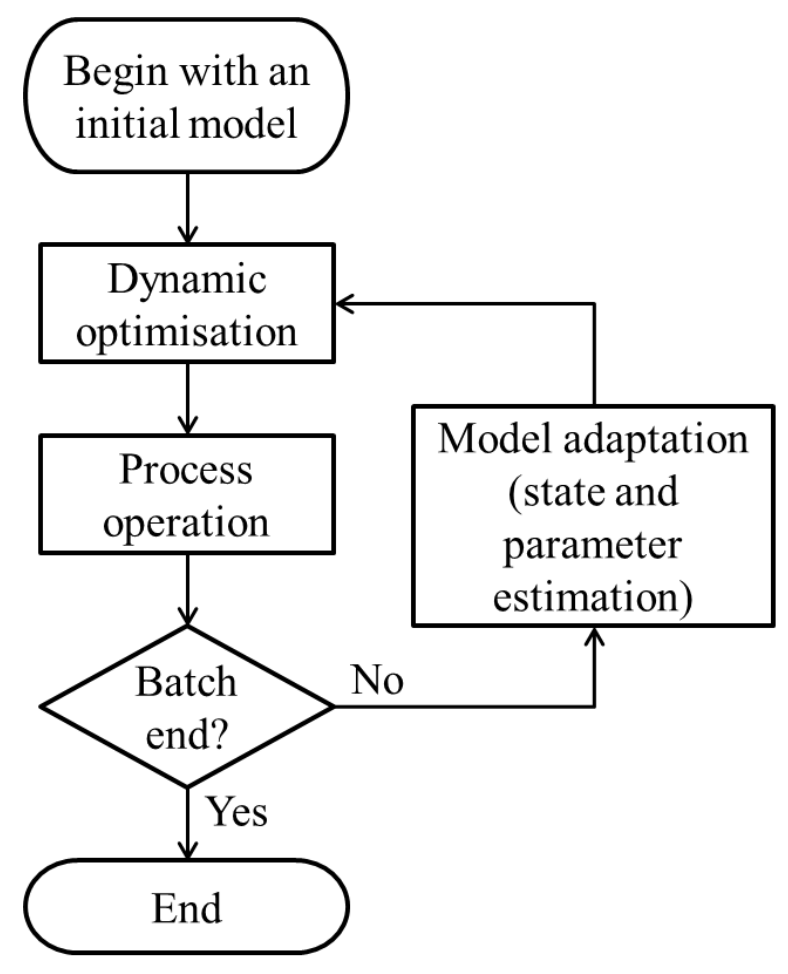

Figure 1: The flow chart of the overall approach. 
(a)



(b)

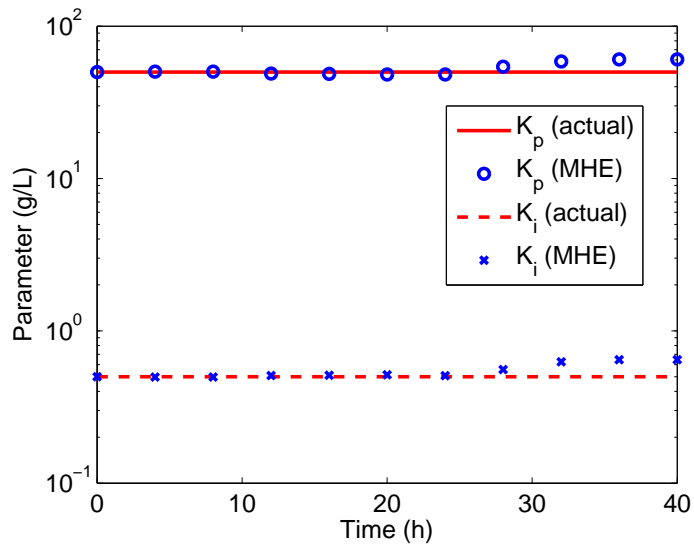

(c)

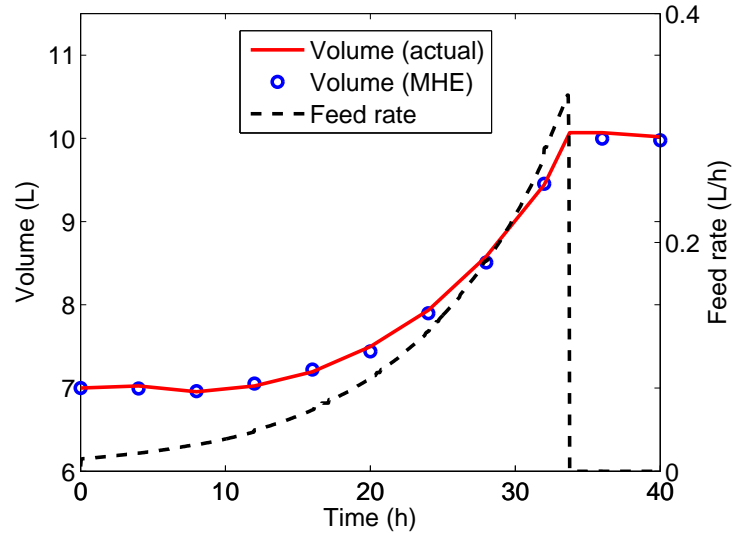

Figure 2: The process trajectory and state/parameter estimation results for the case $r=1.0$, i.e. no initial parameter discrepancy. The weighted objective function with $\alpha=10^{-20}$ was used for optimisation, numerically equivalent to no active adaptation. The end-batch production was $327.7 \mathrm{~g}$. (a) Substrate $(S)$ and biomass $(X)$ concentration; (b) Parameter estimation; (c) Reactor volume and feed rate. 
(a)

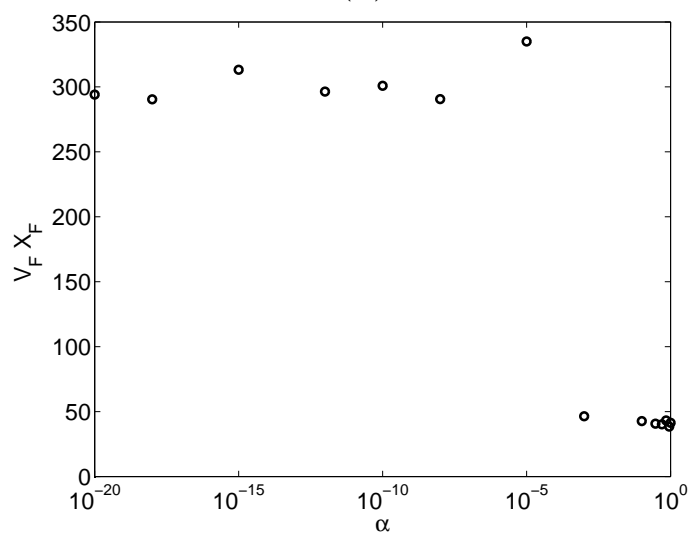

(c)

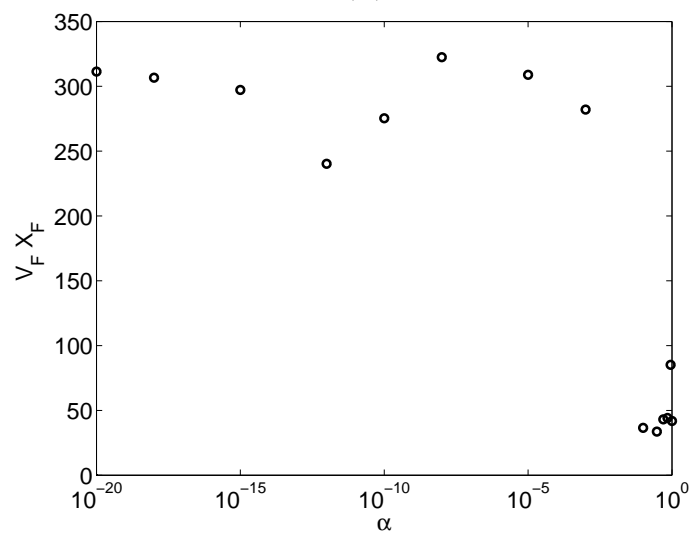

(e)

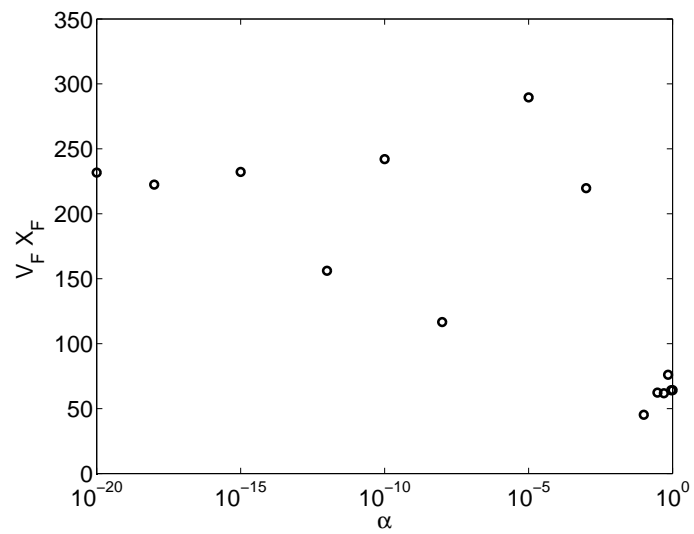

(b)

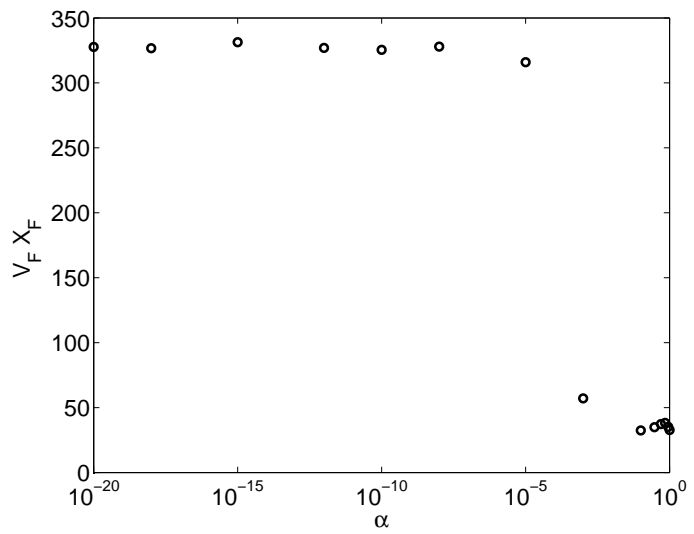

(d)

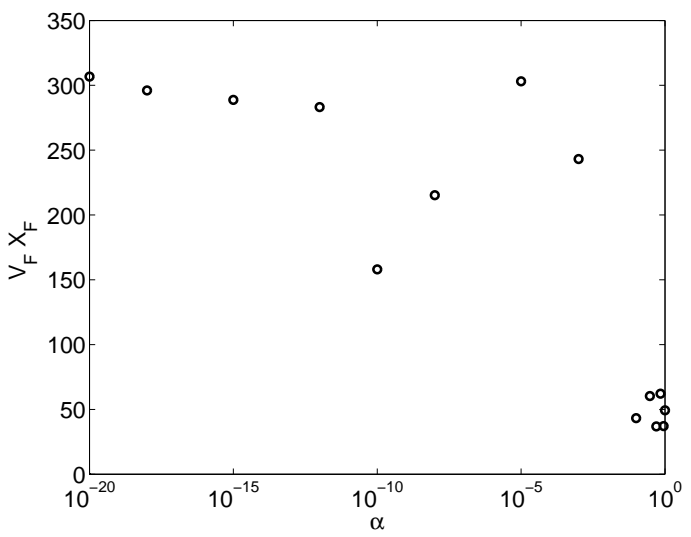

(f)

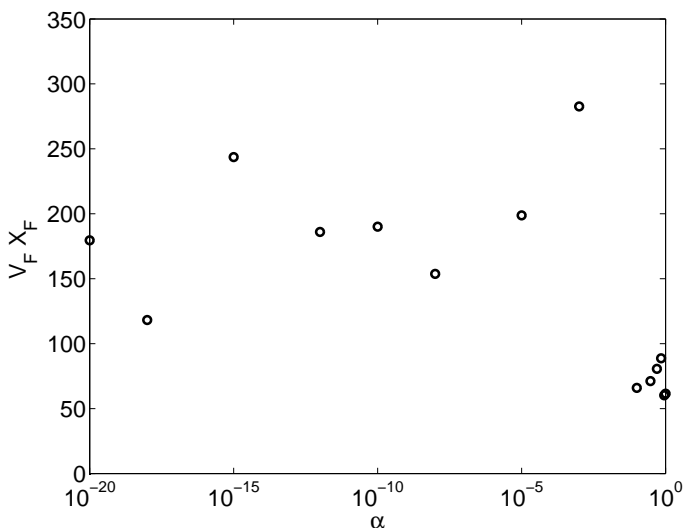

Figure 3: The end-batch biomass production $V_{F} X_{F}$ as a function of the weighting factor $\alpha$, subjecting to initial parameter discrepancy in terms of the multiplier $r$ : (a) $r=0.5$, (b) $r=1.0$, (c) $r=1.5$, (d) $r=2.0$, (e) $r=2.5$, (f) $r=3.0$. 
(a)

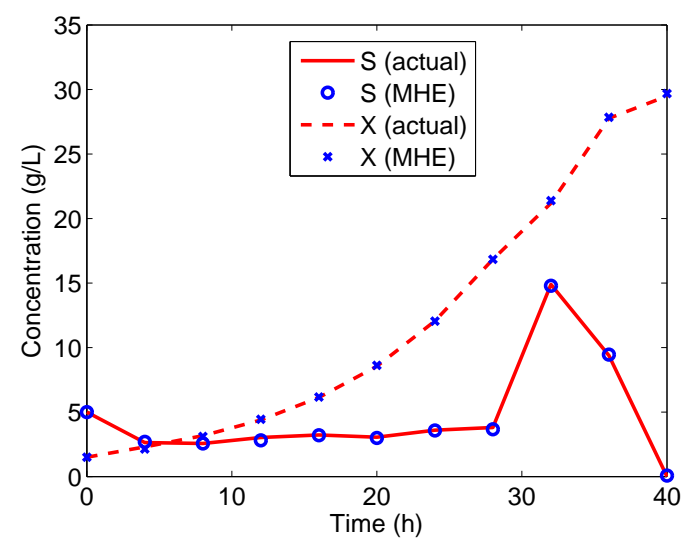

(c)

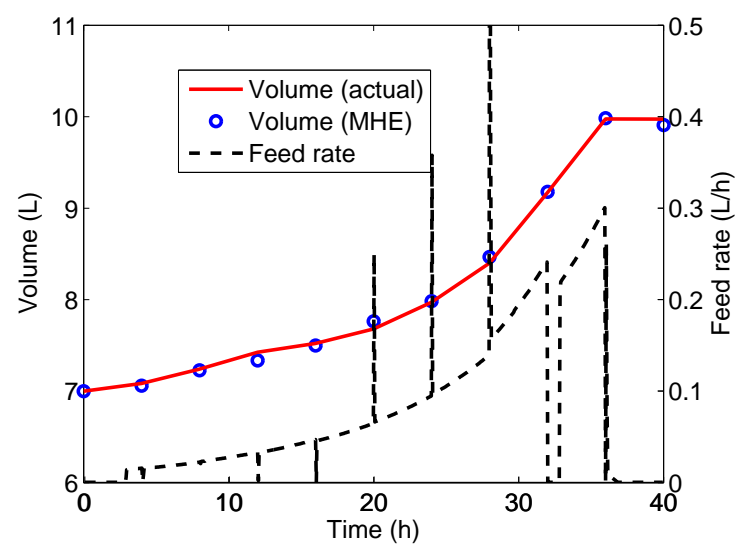

(b)

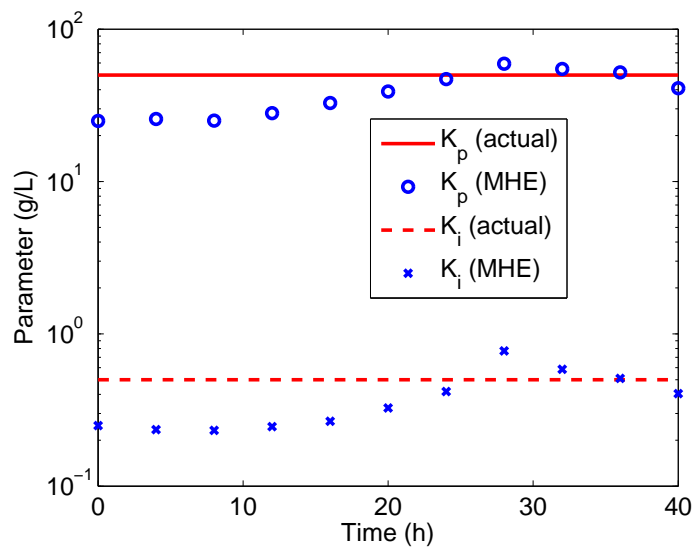

(d)

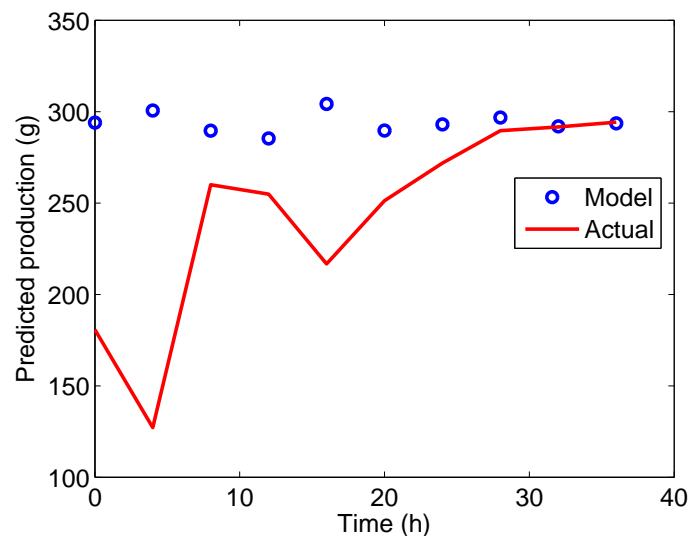

Figure 4: The process trajectory and state/parameter estimation results for the case $r=0.5$. The weighted objective function with $\alpha=10^{-20}$ was used for optimisation, numerically equivalent to no active adaptation. The end-batch production was $294.0 \mathrm{~g}$. (a) Substrate $(S)$ and biomass $(X)$ concentration; (b) Parameter estimation; (c) Reactor volume and feed rate; d) Predicted end-batch production at each sampling interval. 
(a)

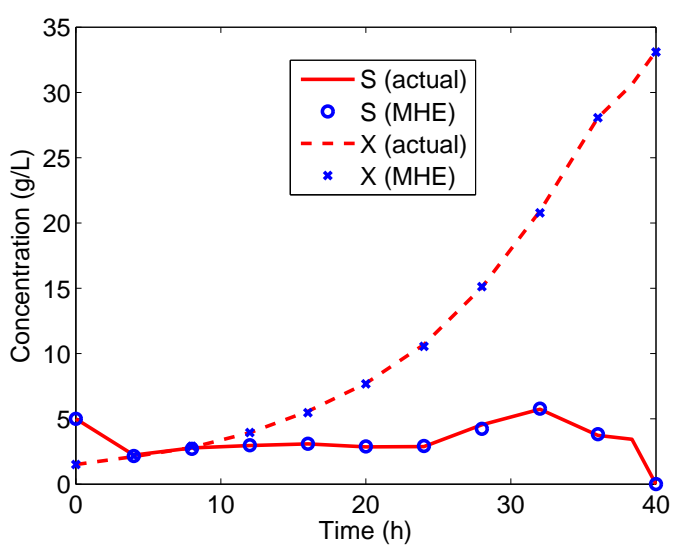

(c)

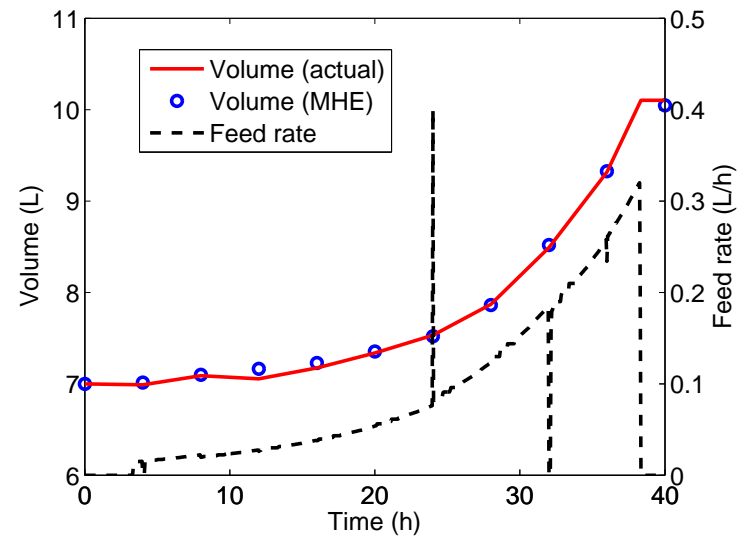

(b)

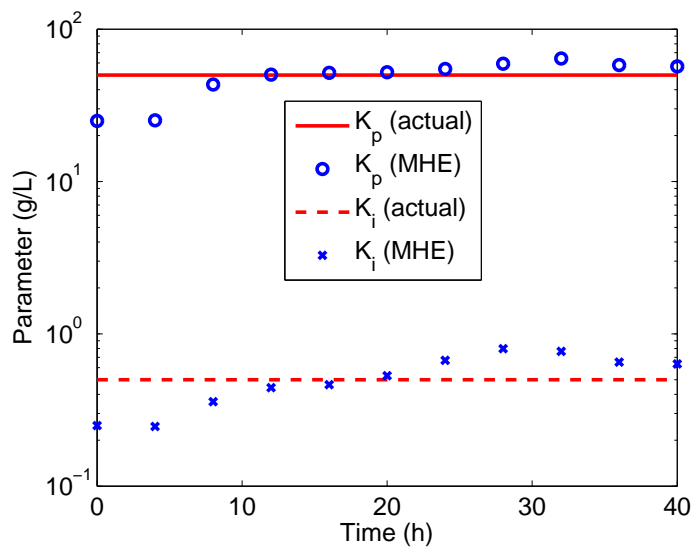

(d)

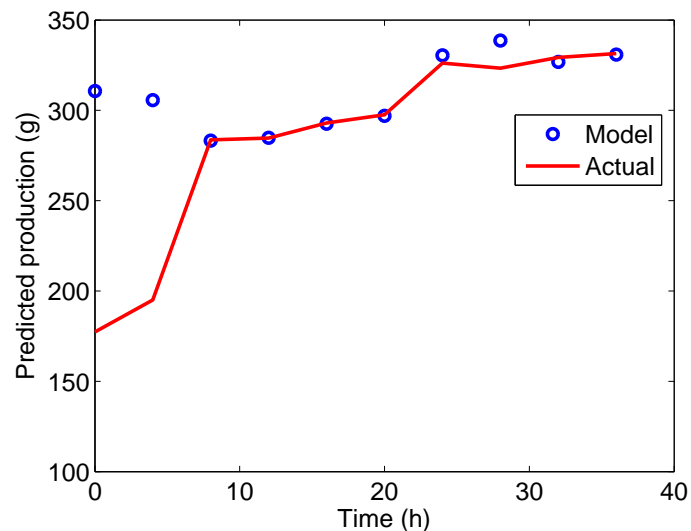

Figure 5: The process trajectory and state/parameter estimation results for the case $r=0.5$. The weighted objective function with $\alpha=10^{-5}$ was used for optimisation, and the end-batch production was 334.9 g. (a) Substrate $(S)$ and biomass $(X)$ concentration; (b) Parameter estimation; (c) Reactor volume and feed rate; (d) Predicted end-batch production at each sampling interval. 
(a)



(c)

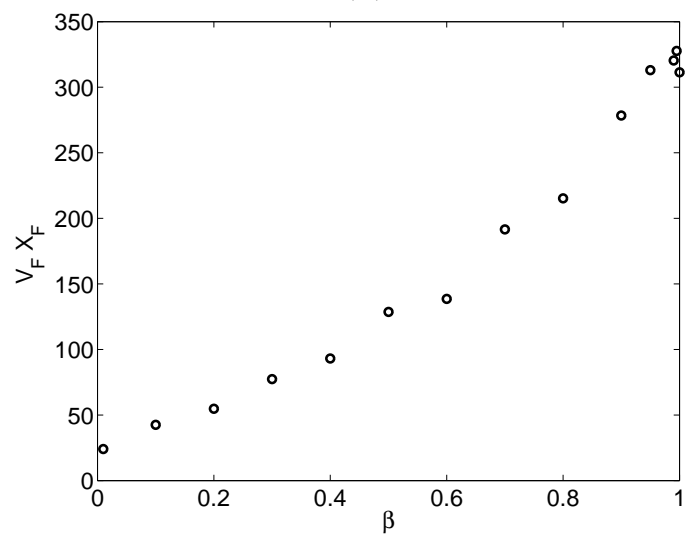

(e)

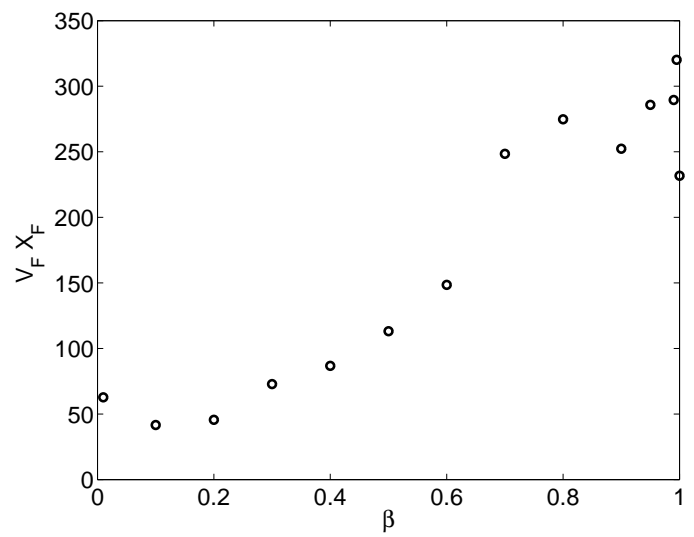

(b)

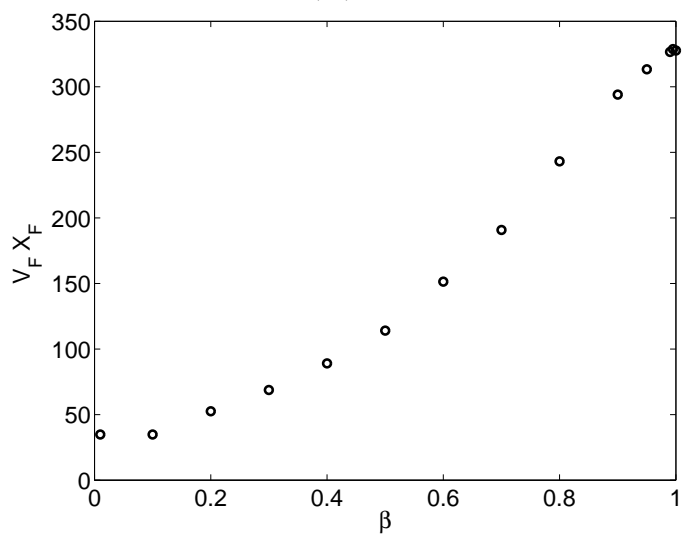

(d)



(f)

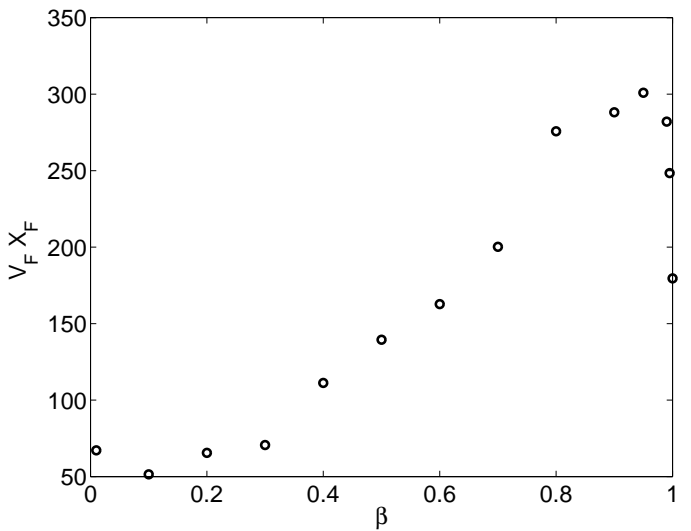

Figure 6: The end-batch biomass production $V_{F} X_{F}$ as a function of the constraint fraction $\beta$, subjecting to initial parameter discrepancy in terms of the multiplier $r$ : (a) $r=0.5$, (b) $r=1.0$, (c) $r=1.5$, (d) $r=2.0$, (e) $r=2.5$, (f) $r=3.0$. 